EPJ Web of Conferences 16, 06002 (2011)

DOI: $10.1051 /$ epjconf/20111606002

(C) Owned by the authors, published by EDP Sciences, 2011

\title{
Understanding sub-stellar populations using wide-field infrared surveys
}

\author{
D.J. Pinfield ${ }^{1, a}$, A.C. Day-Jones ${ }^{1,2}$, B. Burningham ${ }^{1}$, S.K. Leggett ${ }^{3}$, \\ H. Beaumont ${ }^{1}$, M. Tamura ${ }^{4}$, C.G. Tinney ${ }^{5}$, M.C. Liu ${ }^{6}$, D. Homeier ${ }^{7}$, N. Lodieu ${ }^{8}$, \\ N.R. Deacon ${ }^{6}$, A.A. West ${ }^{9}$, N. Huelamo ${ }^{10}$, T. Dupuy ${ }^{6}$, D.J. Mortlock ${ }^{11}$, \\ S.J. Warren ${ }^{11}$, H.R.A. Jones ${ }^{1}$, P.W. Lucas ${ }^{1}$, M. Ishi' ${ }^{12}$, R.G. McMahon ${ }^{13}$, \\ P.C. Hewett ${ }^{13}$, M.R. Zapatero-Osorio ${ }^{10}$, E.L. Martin ${ }^{10}$, B.P. Venemans ${ }^{11}$, \\ D. Barrado ${ }^{10}$, Z. Zhang ${ }^{1}$ and M. Morales-Calderon ${ }^{10}$
}

1 Centre for Astrophysics Research, University of Hertfordshire, Hatfield AL10 9AB, UK

2 Department of Astronomy, Universidad de Chile, Casilla 36-D, Santiago, Chile

3 Gemini Observatory, 670 N. A'ohoku Place, Hilo, HI 96720, USA

${ }^{4}$ National Astronomical Observatory, Mitaka, Tokyo 181-8588, Japan

5 University of New South Wales, Sydney, NSW 2052, Australia

6 Institute for Astronomy, University of Hawai'i, 2680 Woodlawn Drive, Honolulu, HI 96822, USA

7 Georg-August-Universitat, Friedrich-Hund-Platz 1, 37077 Gottingen, Germany

8 Instituto de Astrofisica de Canarias, 38200 La Laguna, Spain

${ }^{9}$ MIT Kavli Institute, 77 Massachusetts Avenue, Cambridge, MA 02139, USA

10 Laboratorio de Astrofisica Estelar y Exoplanetas (LAEX), Spain

11 Imperial College London, Blackett Laboratory, Prince Consort Road, London SW7 2AZ, UK

12 Subaru Telescope, 650 North A'ohoku Place, Hilo, HI 96720, USA

13 Institute of Astronomy, Madingley Road, Cambridge CB3 OHA, UK

\begin{abstract}
This paper discusses benchmark brown dwarfs in various environments, and focuses on those in wide binary systems. We present a summary of the recently discovered $\mathrm{T}$ dwarf population from the UKIDSS Large Area Survey, and describe the constraints that it places on our knowledge of the sub-stellar initial mass function. We also present some exciting results from our ongoing search for wide companions to this sample, that has so far revealed an M4-T8.5 binary system at $\sim 12$ parsecs and also the first ever Tdwarf-white dwarf binary system. The T dwarfs in these binaries have their properties constrained by the primary object and are thus benchmark objects that are already testing the predictions of theoretical model atmospheres.
\end{abstract}

\section{BENCHMARK BROWN DWARFS}

Sub-stellar objects (brown dwarfs and extra-solar planets) are shrouded with complex ultracool atmospheres, and it is an ongoing and major challenge to understand the physics of these environments. However, only a proper understanding of this physics will facilitate future detailed studies of such objects powered by observation, and critical to this is the discovery of benchmark objects whose properties can be well constrained independently.

ae-mail: D.J.Pinfield@herts.ac.uk

This is an Open Access article distributed under the terms of the Creative Commons Attribution-Noncommercial License 3.0, which permits unrestricted use, distribution, and reproduction in any noncommercial medium, provided the original work is properly cited. 


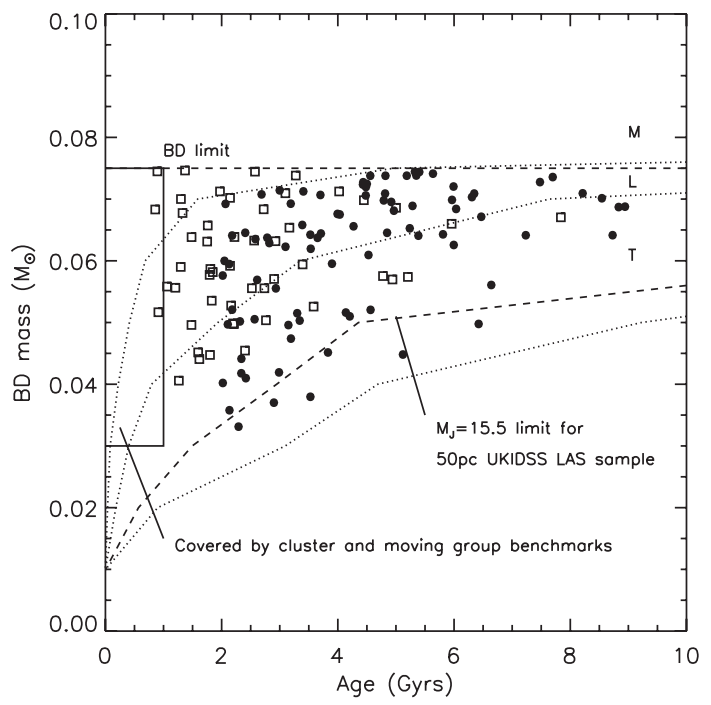

Figure 1. Simulated populations of benchmark brown dwarfs.

Benchmark brown dwarfs are those whose physical properties can be constrained in ways that are relatively independent of theoretical model fits to brown dwarf observables (i.e. spectra and photometry). Examples of benchmark brown dwarfs include;

- Brown dwarfs in open clusters and moving groups - known age and composition, but are all young.

- Tight double brown dwarf binaries - dynamical masses, and thus evolutionary ages.

- Brown dwarfs in eclipsing binary systems - radii.

- Brown dwarf companions at close separation - can be challenging to study.

- Brown dwarf companions at wide separation - easily studied but benchmark quality dependent on the nature of the primary.

Of these, only the brown dwarf companions at wide separation offer the possibility of having a range of useful constraints on their physical properties, spanning a large range of age and metallicity in the Galactic disk. In addition, studies by Pinfield et al. (2006) and Gizis et al. (2001) show that there are a significant fraction of main sequence stars with wide brown dwarf companions - a 2-3\% L dwarf binary fraction for separations $>1000$ AU which could lead to a total brown dwarf binary fraction of 20-30\% (if the companion mass function has $\alpha \sim 1$ ).

As Pinfield et al. (2006) explains, optimal primaries for such benchmark systems would be subgiant stars which can provide accurate age and metallicity constraints, and white dwarfs which can give robust lower limits to age, and in the case of high mass white dwarfs (for which the main sequence progenitor life-time is short) solid age constraints at the $\sim 10 \%$ level. Simulations presented in Pinfield et al. (2006) show that we expect sizable populations of these benchmark systems to be available in new and nearfuture large scale optical and infrared surveys; e.g. the UKIRT Infrared Deep Sky Survey (UKIDSS), the VISTA surveys and the Sloan Digital Sky Survey (SDSS). Figure one shows the expected mass-age distribution of the predicted $\sim 100-150$ benchmark $\mathrm{L}$ and $\mathrm{T}$ type brown dwarfs from these simulations.

\section{SEARCHING FOR T AND COOLER DWARFS IN THE UKIDSS LARGE AREA SURVEY}

To exploit the UKIDSS Large Area Survey (LAS) for T dwarfs we searched the LAS and SDSS sky for objects with colours consistent with observed late $\mathrm{T}$ populations and/or model atmosphere predictions for cooler $T_{\mathrm{eff}} \mathrm{S}$. We assess in the main Y-J and J-H colours, where in some cases we only place upper limits on the J-H colours (i.e. these are YJ-only detected objects with the H-band limits being consistent 
Research, Science and Technology of Brown Dwarfs and Exoplanets
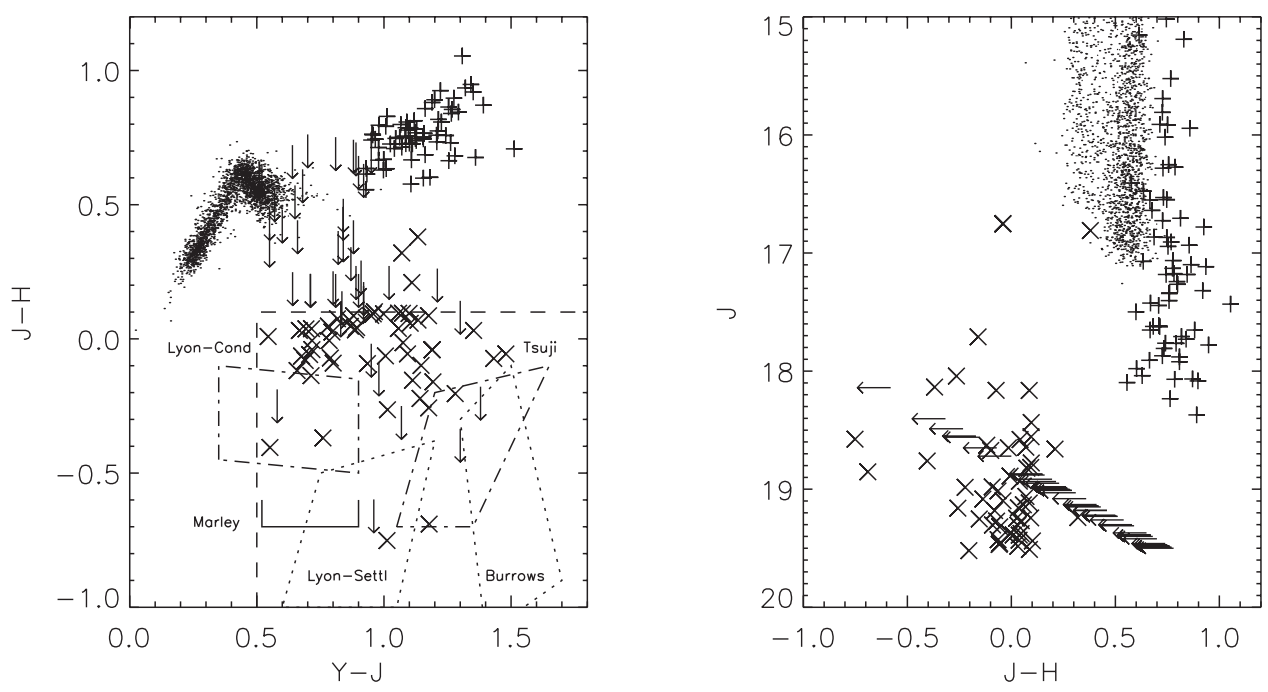

Figure 2. A J-H against $\mathrm{Y}-\mathrm{J}$ two-colour diagram and a $\mathrm{J}$ against $\mathrm{J}-\mathrm{H}$ colour magnitude diagram showing candidate late $\mathrm{T}$ and $\mathrm{Y}$ dwarfs from the UKIDSS LAS. $\mathrm{YJH}(\mathrm{K})$ candidates are shown as crosses, and YJ-only candidates as upper limit arrows. An illustrative L dwarf candidate sample is shown by plus signs. Our YJH candidate selection box is shown with a dashed line, and contains theoretical model predicted colours for Teff $=400-700 \mathrm{~K}$ dwarfs from : the cloud-free Lyon-COND models (Allard et al. 2001; Baraffe et al. 2003); the more recent Lyon-Settl models (Allard et al., in preparation); the AMES models (e.g. Marley et al. 2002; Saumon et al. 2003); the Tucson models (e.g. Burrows et al. 2006) and the Tsuji models (Tsuji et al. 2004).

with blue J-H colour). Figure 2 shows as an example a selection of our candidates (from the second main LAS data release DR2). Several subsequent data releases (currently on DR5) have brought the total LAS coverage to $\sim 2000$ sq degs.

Our follow-up procedure is to initially re-measure the photometry of candidates so as to rule out various forms of contamination - mainly (i) $\mathrm{M}$ dwarfs which can be optical non detections but whose colours have scattered to the blue due to photometric uncertainties, (ii) solar-system objects which can be HK non detections due to motion between different UKIDSS observing nights, and (iii) red galaxies that may contaminate our colour selections. This follow-up imaging is generally carried out $\sim$ a year after the original LAS imaging was done, and it thus also provides us with second epoch images that allow us to measure proper motions for many of our new discoveries.

Good photometric candidates are then measured spectroscopically in the near infrared using $8 \mathrm{~m}$ telescopes. Our programme makes use of GNIRS and NIRI on Gemini, and IRCS on Subaru. We measure $\mathbf{J}$ or $\mathrm{JH}$ spectra to confirm (or otherwise) a T spectral type, and if the object is very late we subsequently obtain higher signal-to-noise JHK spectral coverage. For confirmed late T dwarfs we also have a Spitzer follow-up programme for mid-infrared spectroscopy and photometry, and a $\mathrm{T}$ dwarf parallax programme to measure distances.

\section{THE GROWING LAS T DWARF POPULATION}

Spectral typing of our sample is done using the spectral classification scheme of Burgasser et al. (2006). The spectral type J-magnitude distribution of the sample of LAS T dwarfs identified by our programme (see; Kendall et al. (2007), Lodieu et al. (2007), Pinfield et al. (2008), Lodieu et al. (2009), Burningham et al. (2009c)) shows that we are reasonably sensitive to $\mathrm{T}$ dwarfs with types $\geq \mathrm{T} 4$ and $\mathrm{J}<19$, and have a good level of completion for types $\geq \mathrm{T} 5$ and $\mathrm{J}<18.8$. 


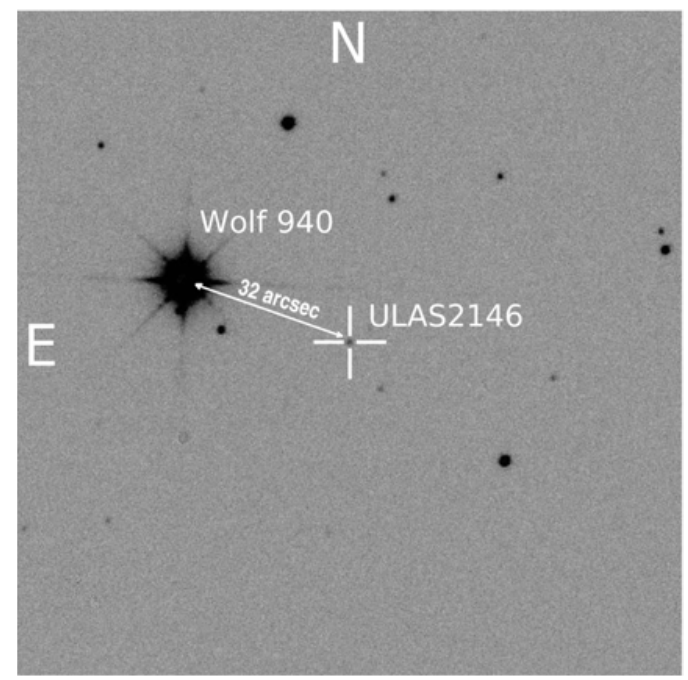

Figure 3. A J-band finder chart image of the T dwarf ULAS2146 (Wolf 940B) and its common proper motion M4 primary Wolf940A.

\section{T8+ DWARFS}

Our programme is identifying a new population of dwarfs with spectral types $>\mathrm{T} 8$, that were previously unknown prior to UKIDSS capabilities. These new objects share some general characteristics as follows: Their measured values of near-infrared absorption spectral indices imply a classification later than T8. Their H-[4.49] colours are significantly redder than for earlier T types. Their J- and H-band spectral peaks are somewhat narrower than T8 dwarfs, with spectral models supporting this as a trend towards lower $T_{\text {eff }}$. At time of writing there are six T8+ dwarfs known (Warren et al. (2007), Burningham et al. (2008), Delorme et al. (2008), Leggett et al. (2009), Burningham et al. (2009a) and Burningham et al. (2009b \& c in press).

\section{T DWARF POPULATION CONSTRAINTS}

By accounting for the main sources of incompleteness (selection, follow-up and spatial) as well as the effects of unresolved binarity, Malmquist and Eddington bias, the number of T dwarfs in our sample is most consistent with theoretical predictions if the substellar mass function exponent $\alpha\left(\mathrm{dN} / \mathrm{dm} \propto m^{-\alpha}\right)$ lies between -1.0 and 0 . This is significantly lower than the $\alpha \sim 0.5-1.0$ suggested by L dwarf field populations and young clusters, which is possibly a result of the lower mass range probed by the T dwarf class.

\section{THE COOLEST BENCHMARK YET - WOLF 940B}

A comparison of old schmidt plate images and the new LAS images of the T dwarf ULAS 2146 revealed a high proper motion M4 star (Wolf 940) 30 arcseconds away on the sky (see Figure 3). Subsequent proper motion measurement of the T dwarf (using the LAS image and one of our near infrared followup photometry images, as first and second epochs respectively) showed that this M4-T8.5 pair are a common proper motion system, and must be a gravitationally bound binary (statistical arguments show that we would expect, by random chance, only $10^{-3}$ spurious common proper motion companions to the entire known LAS T8+ dwarf population). The T dwarf was thus named Wolf 940B. 


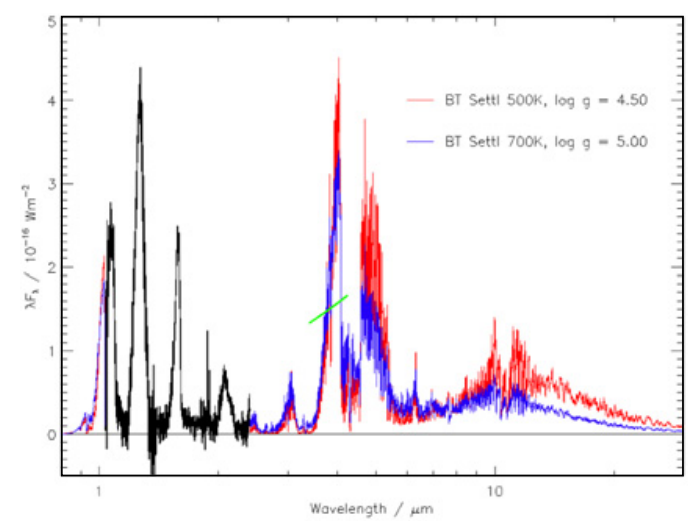

Figure 4. Red to mid-infrared spectrum of the T dwarf ULAS 2146 (Wolf 940B). The red to near infrared spectra are observed and normalised via photometry. The mid infrared region is made up of theoretical spectra that are normalised with measured L-band photometry. The resulting S.E.D. leads to a robust bolometric flux constraint for this T dwarf.

The parallax of Wolf 940A is accurately known, and the distance of the T dwarf can thus be inferred as $12.5_{0.67}^{0.25} \mathrm{pc}$. This distance constraint reveals that Wolf $940 \mathrm{~B}$ is one of the faintest T dwarfs ever found.

In addition, adaptive optics imaging with the Keck laser-guide-star system has shown that Wolf 940B is likely to be a single object rather than an unresolved binary brown dwarf. As a single object we can relate its luminosity directly to its $T_{\text {eff }}$ and radii (where $\mathrm{T}$ dwarf radii can be robustly assumed from evolutionary models).

Combined red-mid infrared spectroscopy and photometry accurately constrain the bolometric flux of Wolf 940B (see Figure 4), and with its known distance its luminosity is revealed. Reasonable age and compositional constraints from the primary thus lead to evolutionary mass and radii estimates for the $\mathrm{T}$ dwarf, and we have thus determined this benchmark object to have $T_{\text {eff }}=570 \pm 25 \mathrm{~K}, \log \mathrm{g}=4.75-$ 5.00 and $[\mathrm{M} / \mathrm{H}]=-0.06 \pm 0.20$.

The physical constraints on this benchmark object allow it to be used to test theoretical models. Figures 5 and 6 show plots of two spectroscopic properties thought to be sensitive to physical properties in this temperature regime. $\mathrm{W}_{J}$ is a spectral ratio sensitive to the width of the J-band flux peak, and $\mathrm{K} / \mathrm{J}$ is the ratio of the central brightness of the T dwarf's K- and J-band flux peaks. Grids of theoretical model predictions are shown for different $T_{\text {eff }}$ and $\log \mathrm{g}$, and it is clear that the location of Wolf $940 \mathrm{~B}$ in the Figure 5 is inconsistent with the model predictions. Even when the model grids are normalised through use of another benchmark object (in Figure 6), significant differences still remain between the predictions for and observations of Wolf 940B.

\section{THE FIRST T DWARF - WHITE DWARF BENCHMARK}

We have combined UKIDSS LAS images with all available follow-up imaging to measure proper motions of as many of our LAS T dwarf sample as possible. At the time of this conference we have measured proper motions for 17 of our spectroscopically confirmed LAS T dwarfs, and have searched in the SuperCOSMOS Science Archive for possible common proper motion companions out to distances corresponding to 20,000 AU at the estimated distance (Liu et al. 2006) of each T dwarf. We also used the estimated $\mathrm{T}$ dwarf distances to place candidate companions on a colour-magnitude diagram so as to identify possible main sequence and white dwarf companions. The only main sequence companion identified was Wolf 940B (which we had already found serendipitously), but we identified a number of white dwarf candidate companions (see Figure 7). 


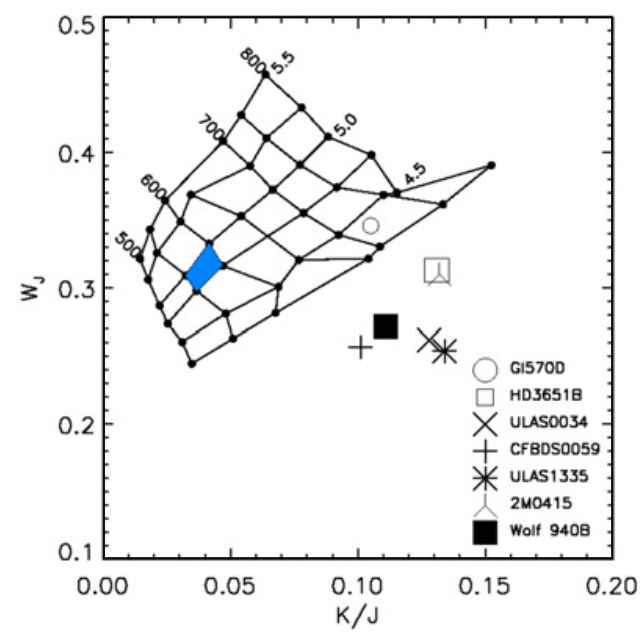

Figure 5. WJ verses K/J indices for a grid of the most recent set of solar metallicity BT-Settl model spectra. Flux ratios for HD 3651B and 2MASS 0415 were measured using the spectra from Burgasser (2007), and for Gl 570D using the spectrum from Geballe et al. (2001). Those for the three T9 dwarfs ULAS 0034, CFBDS 0059 and ULAS 1335 were taken from their respective discovery papers (Warren et al. 2007; Delorme et al. 2008a; Burningham et al. 2008). The blue shading indicates the region of this grid that should contain Wolf 940B. Uncertainties are of similar size to the symbols.

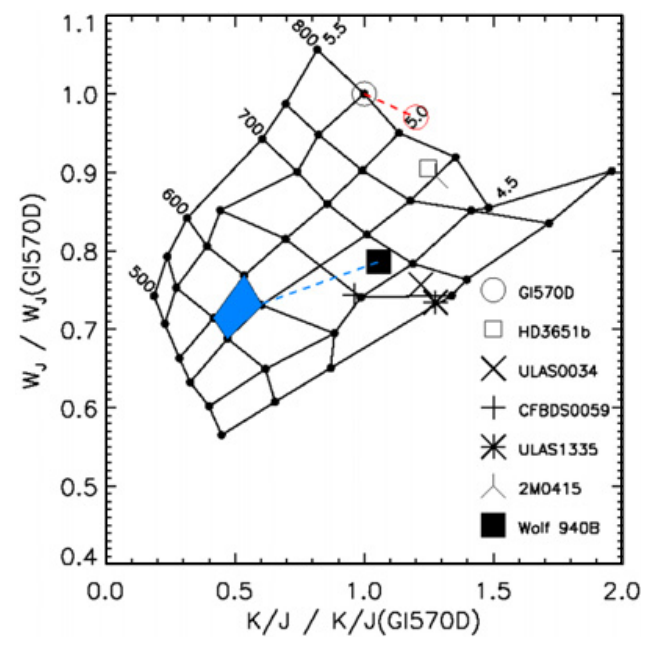

Figure 6. WJ verses $\mathrm{K} / \mathrm{J}$ indices for a grid of solar metallicity BT-Settl model spectra. The grid is normalised such that the model values for the Teff and $\log \mathrm{g}$ of G1570D lie at coordinates $(1,1)$. All observed values for $\mathrm{W}_{J}$ and $\mathrm{K} / \mathrm{J}$ are shown as a proportion of those of GL 570D. We have indicated, with a red dotted line and open circle, the relative shift in position on the grid associated with increasing metallicity by +0.1 dex. Uncertainties are of similar size to the symbols. The blue dotted line highlights the difference between the true properties of Wolf $940 \mathrm{~B}$, and those expected from the model grid.

We then compared the colours of these candidates to other stellar populations, and removed object that appeared to be likely $\mathrm{M}$ dwarfs. This left six remaining good white dwarf candidates.

One of these candidate systems (containing a T4.5 dwarf) was followed up by targeting the white dwarf candidate component using FORS on the VLT. The FORS spectrum is shown in Figure 8, and apart from some telluric features that remain in the spectrum, the object shows essentially a black body morphology with a weak H-alpha line. 


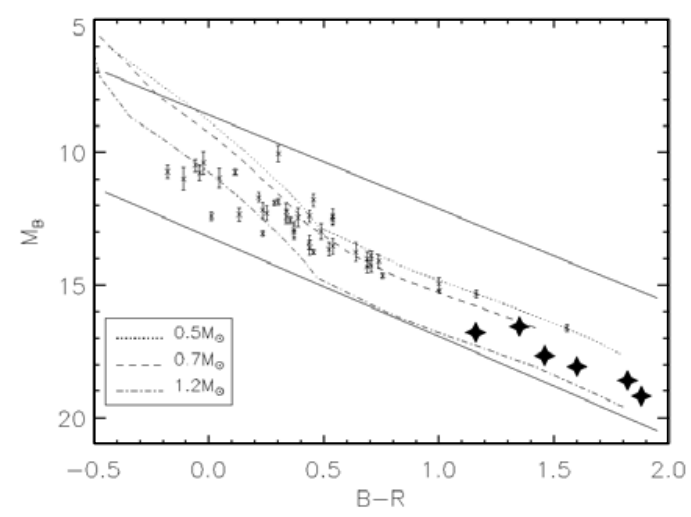

Figure 7. The colour-magnitude white dwarf candidate selection plot, where white dwarf candidate distances have been inferred from the distance constraints on their associated $\mathrm{T}$ dwarf candidate companions.

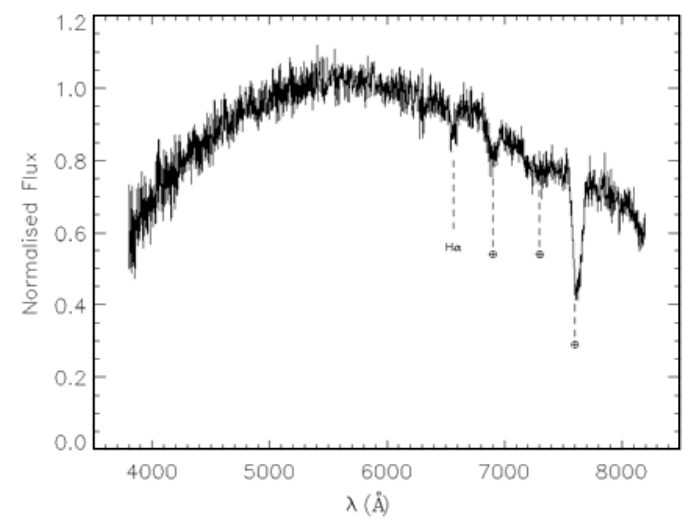

Figure 8. The VLT optical spectrum of the white dwarf component of the T dwarf - white dwarf binary system.

The companion candidate was thus confirmed as a white dwarf, and statistical arguments demonstrate that we would need to search around about one hundred times as many LAS T dwarfs before we found one such companion that was spurious, with common proper motion by random chance. The system is thus statistically shown to be a genuine binary system, and the components thus have a common age (are coeval). A J-band finder chart is shown in Figure 9.

Fits to the SED of the white dwarf provide constraints on its mass and age, and when we also account for the potential lifetime of its main sequence progenitor we estimate a minimum age of the binary system to be 4 Gyrs.

We can infer this age for the T dwarf also, and conclude that it is a fully contracted object, with $T_{\text {eff }}=1200-1500 \mathrm{~K}$ (making use of the $T_{\text {eff }}-$ spectral type relation of Golimowski et al. 2004). COND isochrones for these $T_{\text {eff }}$ and age constraints lead to surface gravity constraints of $\log g=5.42-5.49$. This is thus a typical example of an old-disk T dwarf with relatively high gravity (a typical young-disk $\mathrm{T}$ dwarf would be expected to have a surface gravity of $\log g=5.0$ ).

\section{CONCLUSIONS}

It is clear that the new generation of large scale deep optical and near infrared surveys are revealing large populations of substellar objects. The statistical power of the new samples is shedding new light on the 


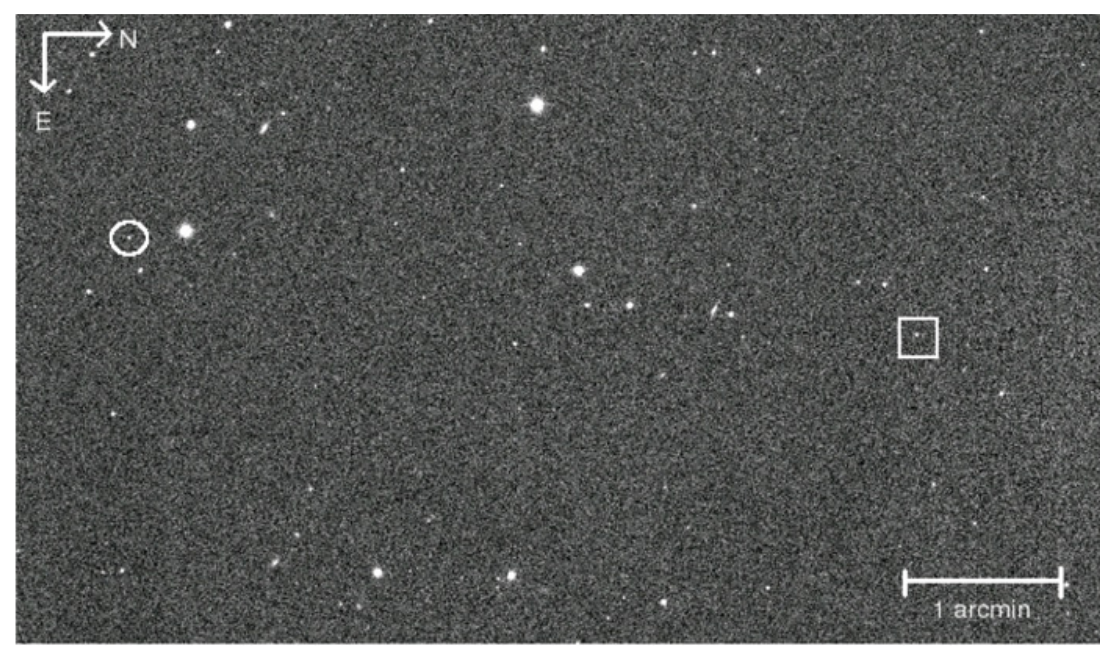

Figure 9. A J-band finder chart image of the T dwarf - white dwarf binary system, indicated by a square and a circle respectively.

substellar initial mass function, as well as probing new temperature ranges below what had previously been explored by surveys like 2MASS.

It is also clear that a number of these sub-stellar objects are members of a variety of wide binary system. Studies of these systems are placing constraints on the physical properties of the substellar components, and these benchmark objects are thus becoming powerful tools for high-lighting differences between atmospheric model predictions and observation.

As our sample of benchmark objects increases in size and fills a wider range of $T_{\text {eff }} / \log \mathrm{g} /[\mathrm{M} / \mathrm{H}]$ parameter space it will become possible to rigorously test the models, and potentially establish an observationally driven means of calibrating the properties of substellar objects.

\section{References}

[1] Burgasser, A.J. et al., ApJ, 658, (2007) 617

[2] Burgasser, A.J. et al., ApJ, 637, (2006) 1067

[3] Burningham, B. et al., MNRAS, 391, (2008) 320

[4] Burningham, B. et al., MNRAS, 395, (2009) 1237

[5] Delorme, P. et al., A\&A, 482, (2008) 961

[6] Geballe, T. R. et al., ApJ, 556, (2001) 373

[7] Gizis, J. et al., ApJL, 551, (2001) 163

[8] Golimowski, D. et al., AJ, 127, (2004) 3516

[9] Kendall, T. et al., A\&A, 466, (2007) 1059

[10] Leggett, S. K. et al., ApJ, 695, (2009) 1517

[11] Liu, M. et al., ApJ, 647, (2006) 1393

[12] Lodieu, N. et al., MNRAS, 379, (2007) 1423

[13] Lodieu, N. et al., MNRAS, 397, (2007) 258

[14] Pinfield, D. J. et al., MNRAS, 368, (2006) 1281

[15] Pinfield, D. J. et al., MNRAS, 390, (2008) 304

[16] Warren, S. J. et al., MNRAS, 381, (2007) 1400 